\title{
SUBSURFACE MELTING OF NYLON BY FRICTION-INDUCED VIBRATIONS*
}

\author{
P. H. VROEGOP and R. BOSMA
}

Twente University of Technology, Department of Mechanical Engineering, Tribology Section, P.O. Box 217, 7500 AE Enschede (The Netherlands)

(Received April 9, 1985; accepted May 9, 1985)

\section{Summary}

Dry sliding of nylon on steel may lead to subsurface recrystallization of the polymer. This phenomenon is described and explained by subsurface melting due to internal heating as a result of the dissipation of frictioninduced vibrations at frequencies above $10 \mathrm{kHz}$. A vibration model relating the distribution of stress and temperature is proposed. Calculations based on this model show reasonable agreement with experimental observations.

\section{Introduction}

During the investigation of friction and wear of polymers on polymers or on other materials, a multitude of strange and still-unexplained phenomena can be observed, reflecting the complicated and often stochastic nature of interaction processes between solid surfaces. In this paper two of these phenomena, observed during sliding of different types of nylon on steel, are described and related.

The first phenomenon is the formation of a protrusion that sometimes occurs at the side of the nylon slider, somewhat below the wear surface. As can be seen from Fig. 1, this phenomenon exists for different geometries and actually was observed on different test rigs. To the present authors' knowledge it has not been reported before.

The second phenomenon is the occurrence of vibrations at rather high frequencies. Friction-induced vibrations have been studied for more than 100 years [1 - 3], although the notion of the relation between friction and vibrations (or rather between sound and the relative motion of bodies) is much older [4]. These vibrations are caused by a continuous or abrupt decrease in friction with increasing specd of sliding, introducing a negative damping in the system. The waveform of these vibrations is a complicated

*Paper presented at the International Conference on Wear of Materials, Vancouver, Canada, April $14 \cdot 18,1985$. 


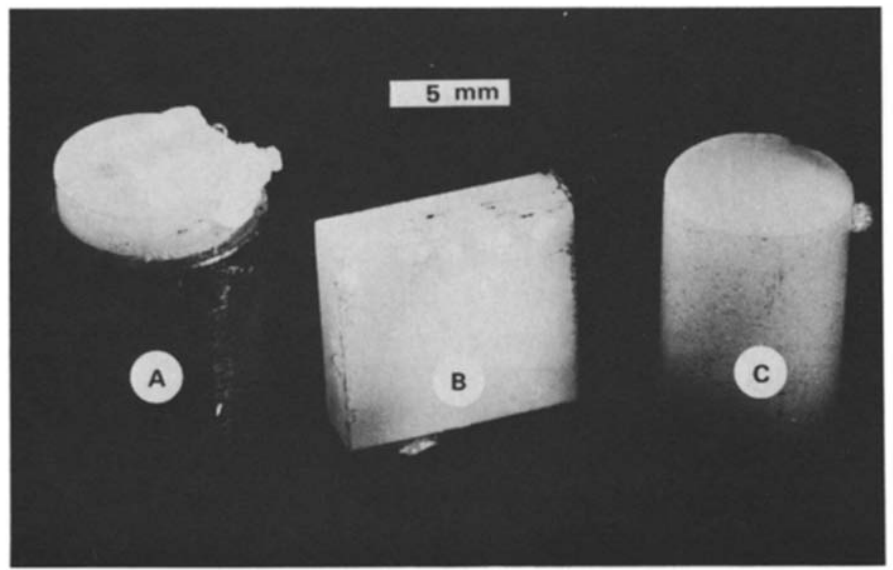

Fig. 1. Polymer specimens showing extruded melt tested on various machines: A, PS II tribometer; B, S tribometer; C, pin-and-ring machine. The wear surface is the upper surface.

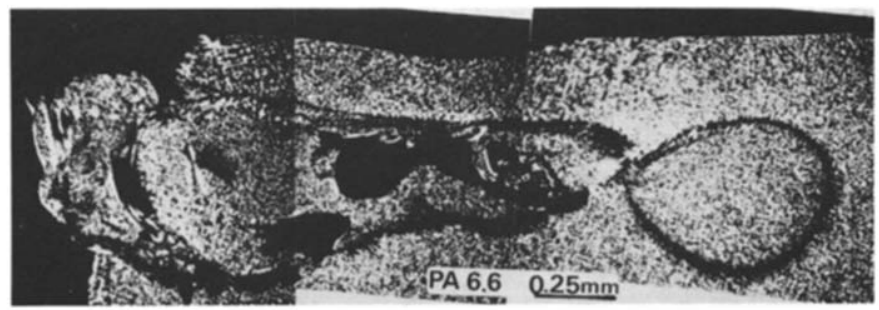

Fig. 2. Thin slice $(5 \mu \mathrm{m})$ of Nylon 6.6 perpendicular to the wear surface showing voids, recrystallization boundaries and extruded melt. The wear surface is the upper surface.

function of the elastic and damping properties of a machine for vibrations below its first natural frequency. For higher frequencies the properties of elements near the source of the vibrations seem to become more important [5].

In this paper a model is proposed to estimate the frequency of vibrations and an explanation is offered for the occurrence of the first phenomenon in terms of subsurface melting due to thermomechanical coupling. Photomicrographs of thin slices perpendicular and parallel to the wear surface (Fig. 2; see also Figs. 11 and 12) clearly show evidence of repeated melting, flow and recrystallization of the nylon. These figures will be discussed in Section 4.

Thermomechanical coupling has been investigated theoretically by several researchers [6]. For the class of vibration problems where the rise in temperature over one cycle is very small, an approximated dissipation function has been derived in terms of the loss modulus and the strain components [7]. This dissipation function applies for the cyclic loading of a linear, homogeneous, isotropic and rheologically simple material. 
For such materials the temperature and stress distributions have been calculated for rods [8], cylinders [9] and plates [10] subjected to harmonic stresses. Essentially the problem consists of simultaneously solving the real and imaginary parts of the wave equation and of the energy equation for a dissipative material with time- and temperature-dependent properties together with an appropriate set of boundary conditions. The main difficulty is to formulate a constitutional equation for the nylon, since the complex modulus and the Poisson constant depend not only on temperature and frequency but also on the amount of water absorbed in the nylon.

\section{Experimental details}

\subsection{Tribometers}

The phenomena of subsurface recrystallization and of vibrations at frequencies above $10 \mathrm{kHz}$ have been observed on three different test rigs with different mechanical, thermal and geometric properties. Most of the data presented here were obtained on a pin-on-disc machine, the PS II tribometer [11]. The temperature of the disc is controlled by a thermostat. The other test rigs were a pin-and-ring machine [12] and a reciprocating rig using a strip-on-plate geometry, the $\mathrm{S}$ tribometer.

The last machine (Fig. 3) basically consists of three parts, i.e. the plate holder, the holder for the rider and the driving system. The plate holder contains a heating and cooling system, controlled by a thermostat, while the friction force is measured on this holder with a piezoelectric force transducer. The rider, a nylon strip, is loaded through a bellows with pressurized air from a thermally isolated vessel. The driving system forces a sinusoidal reciprocating motion (with a stroke of $46 \mathrm{~mm}$ ) parallel to the plate surface on the rider and its loading system.

The dominant natural frequencies of these machines range from less than $100 \mathrm{~Hz}$ for the pin-and-ring machine to $8 \mathrm{kHz}$ for the reciprocating machine. These frequencies are typically determined by the stiffness of the

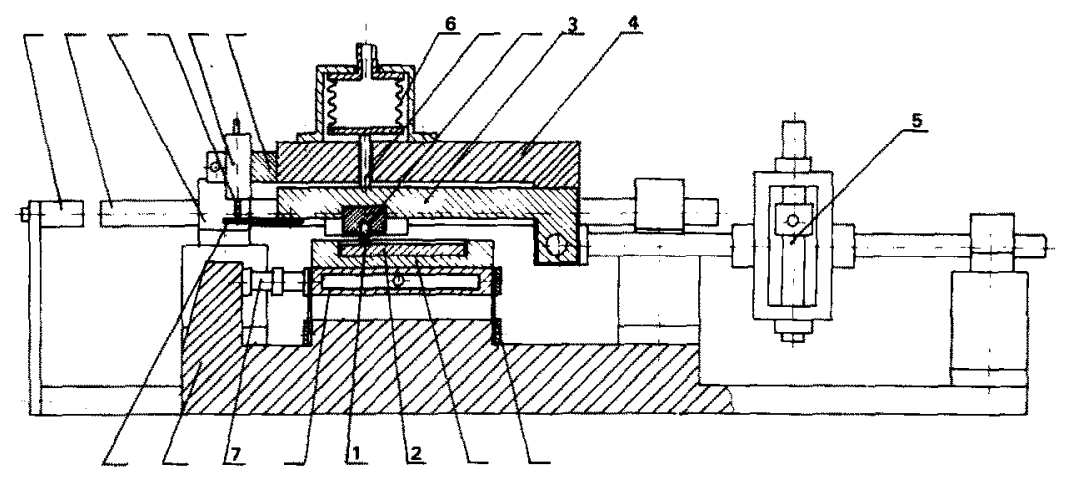

Fig. 3. Schematic drawing of the $\mathbf{S}$ tribometer: 1 , rider; 2 , plate; 3 , loading arm; 4 , reciprocating support; 5 , drive; 6 , bellows; 7 , piezoelectric force transducer. 


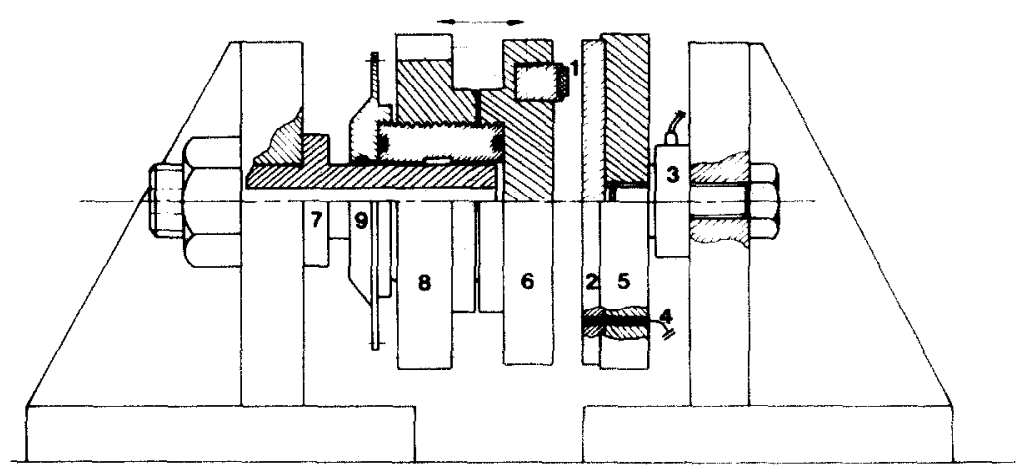

Fig. 4. PS II tribometer: 1, pin; 2, disc; 3, three-component piezoelectric force transducer; 4 , thermocouple; 5 , disc holder with heater and cooling device; 6 , pin holder; 7 , hollow shaft for loading and support of the pin; 8 , gear drive.

force transducer and the mass directly connected to it. For the PS II tribometer (Fig. 4) these frequencies are for translation of the disc and the disc holder between 2 and $4 \mathrm{kHz}$ and for rotation between 0.7 and $1.8 \mathrm{kHz}$.

Vibrations were measured with the piezoelectric force transducers (having natural frequencies greater than $100 \mathrm{kHz}$ ) and with a miniature accelerometer (mass, $15 \mathrm{~g}$ ). The signals were analysed with a real-time spectrum analyser.

\subsection{Materials and operating conditions}

Experiments were performed with different metals ranging from carbon steel to stainless steel. Also different types of nylon were used: Nylon 6, Nylon 4.6 and Nylon 6.6 with and without $\mathrm{MoS}_{2}$ as a filler. The specimens were machined from extruded rods and hot-pressed plates obtained from several manufacturers. The metal surfaces were abraded in random directions to a surface roughness $R_{\mathrm{a}}$ of $0.4 \mu \mathrm{m}$ or less.

Prior to the experiments both specimens were cleaned with carbon tetrachloride in an ultrasonic bath and dried in hot air. Operating conditions on the different tribometers ranged from 0.1 to $4 \mathrm{MPa}(20-100 \mathrm{~N})$ for the normal load, $0.1-1.5 \mathrm{~m} \mathrm{~s}^{-1}$ for the speed of sliding and $20-120{ }^{\circ} \mathrm{C}$ for the mean temperature of the metal surface.

After the experiments, the nylon specimens were cut into thin slices with a microtome to investigate subsurface failure. The specimens are shown in Fig. 1. Specimen $\mathrm{A}$ is glued into a brass holder. This specimen has a thickness of $4 \mathrm{~mm}$ and extends about $3 \mathrm{~mm}$ from the holder. Specimens B and C are clamped in holders leaving a free length of $2 \mathrm{~mm}$ and $4 \mathrm{~mm}$ respectively.

\section{The vibration model}

\subsection{Mathematical model}

Let us consider the shearing of an infinite slab of a viscoelastic material with density $\rho$ and thickness $d$. One surface is bonded, the other surface 
(friction or wear surface) is excited by a sinusoidal shear stress with frequency $\omega$ and amplitude $\tau_{\mathrm{s}}$. Further, let us assume that the temperature on both surfaces is kept constant. In non-dimensionalized form the equation for the stresses can then be written as

$\tau^{\prime \prime}+f^{2} g(\theta) \exp (-\mathrm{i} \delta) \tau=0$

where the complex shear modulus is given as

$G(T, \omega)=\frac{G_{0} \exp (\mathrm{i} \delta)}{g(\theta)}$

and $f$, the non-dimensional frequency, is given as

$f=\omega d\left(\rho / G_{0}\right)^{1 / 2}$

The function $g(\theta)$ contains the time- and temperature-dependent properties. $G_{0}$ is the dynamic modulus at a given temperature and frequency, $\delta$ is the loss angle and $\tau$ is the complex shear stress divided by $\tau_{s}$.

The energy equation can be written as

$\frac{\rho C \dot{\theta}}{\lambda}=\theta^{\prime \prime}+f t^{2} g(\theta) \sin \delta|\tau|^{2}$

where $t$ is the non-dimensional excitation amplitude:

$t=\left|\tau_{\mathrm{s}}\right|\left\{\frac{d}{2 \lambda T_{\mathrm{m}}\left(\rho G_{0}\right)^{1 / 2}}\right\}^{1 / 2}$

The heat conduction and heat capacity are represented by $\lambda$ and $C$, and $\theta$ is the absolute temperature $T$ divided by the melting temperature $T_{\mathrm{m}}$ of the slab.

The boundary conditions are

$\xi=0 \quad \operatorname{Re}\left(\tau^{\prime}\right)=\operatorname{Im}\left(\tau^{\prime}\right)=0 \quad \theta=\theta_{0}$

for the bonded surface and

$\xi=1 \quad \operatorname{Re}(\tau)=1 \quad \operatorname{Im}(\tau)=0 \quad \theta=\theta_{\mathrm{s}}$

for the wear surface. Re and Im denote the real and imaginary parts of a complex variable. The prime and the dot denote differentiation with respect to the depth $\xi$ into the slab and the time respectively.

\subsection{Observations on the model}

The equations shown above present a set of non-linear second-order differential equations coupled by the function $g(\theta)$. Between the glass transition temperature and the melting point the shear modulus for semicrystalline polymers can be described as

$\log \{g(\theta)\}=A \log \left(\frac{\omega_{\mathrm{R}}}{\omega}\right)+B\left(\frac{1}{\theta_{\mathrm{R}}}-\frac{1}{\theta}\right)$ 


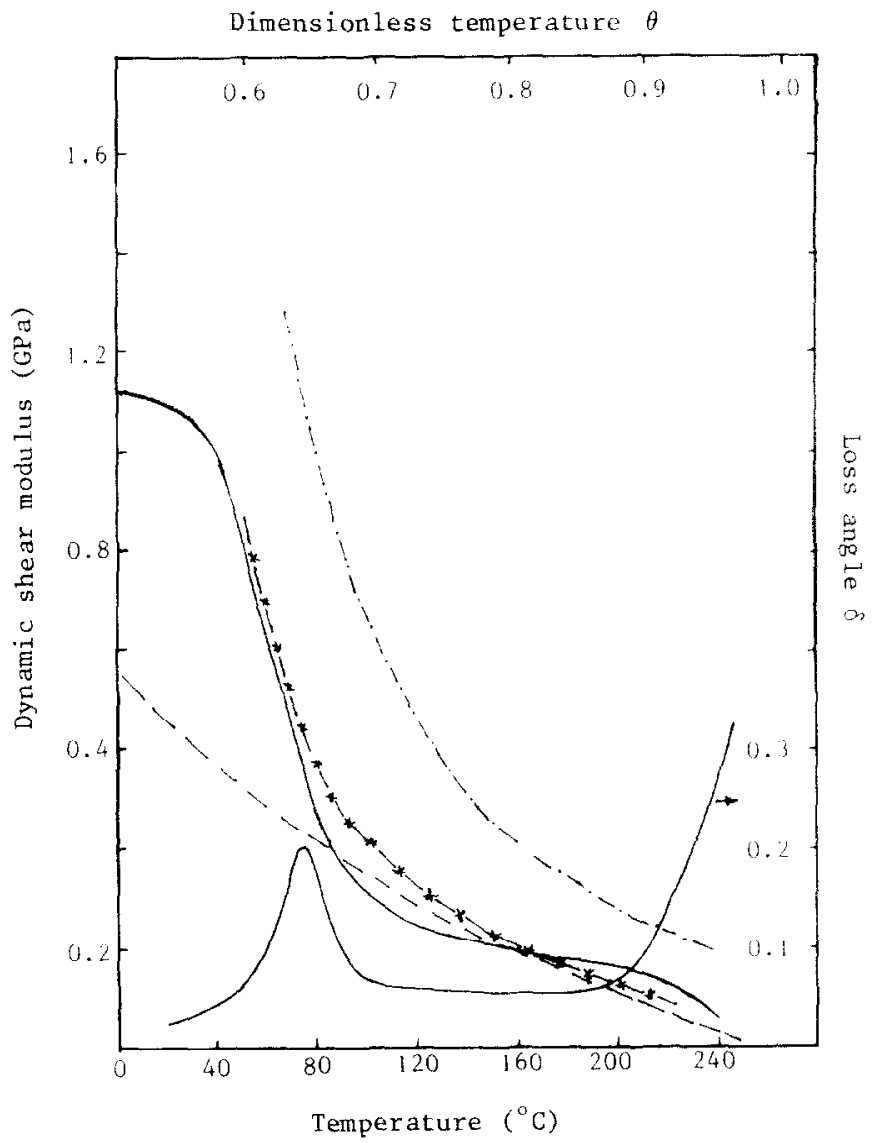

Fig. 5. Dynamic shear modulus of Nylon 6.6 vs. temperature: - - - - calculated, $20 \mathrm{kHz}$, dry; - - - , calculated, $20 \mathrm{kHz}, 65 \%$ relative humidity; - - relation used in numerical calculations. Also shown is the loss angle $\delta$ vs. temperature.

For Nylon 6.6 the constants in this empirical equation can be deduced [13] as $A=0.1, B=1.65, \omega_{\mathrm{R}}=1 \mathrm{~Hz}, \theta_{\mathrm{R}}=0.57$ and $G_{0}=1.3 \mathrm{GPa}$.

The modulus calculated from this relation is plotted in Fig. 5, together with the storage modulus and the loss angle as measured for a dry sample at $1 \mathrm{~Hz}$. The effect of relative humidity [13] is also shown. As can be seen, the influence of absorbed water (or water solubles) is very large. Consequently, for a correct description of the problem, one would have to add another second-order differential equation describing the diffusion and the resultant concentration of water in the slab. Since the heat conductivity, the heat capacity and the density also depend on temperature, it would be a difficult task to solve this problem even if all material properties were known.

\subsection{Approximate solutions}

In this section two solutions are presented. The first solution can be obtained in closed form and assumes all material properties to be constant. 
In the second solution only the modulus varies with temperature, according to the broken curve in Fig. 5. Both solutions assume thermal equilibrium, so the left-hand side of eqn. (4) equals zero.

For constant material properties eqns. (1) and (4) become uncoupled $(g(\theta)=1)$. The distribution of the stress amplitude, satisfying eqn. (1) and the boundary conditions, is

$\tau=\frac{\cos (\gamma \xi)}{\cos \gamma}$

where

$\gamma=f \exp \left(-\frac{\mathrm{i} \delta}{2}\right)$

Substitution of this solution into the energy equation (4) and integrating twice yields the temperature distribution as

$\theta=C\left\{\beta^{2} \cos (2 \alpha \xi)-\alpha^{2} \cosh (2 \beta \xi)\right\}+C_{1} \xi+C_{2}$

where

$\alpha=f \cos (\delta / 2)$

$\beta=f \sin (\delta / 2)$

$C=\frac{t^{2}}{2 \alpha \beta f\{\cos (2 \alpha)+\cosh (2 \beta)\}}$

$C_{1}=\theta_{s}-\theta_{0}+C\left[\beta^{2}-\alpha^{2}-\left\{\beta^{2} \cos (2 \alpha)-\alpha^{2} \cosh (2 \beta)\right\}\right]$

and

$C_{2}=\theta_{0}-C\left(\beta^{2}-\alpha^{2}\right)$

If the condition that the maximum temperature equals the melting temperature $\left(\theta_{\max }=1\right)$ is imposed on this solution, a relation between the frequency $f$ and the amplitude $t$ for given material properties and boundary temperatures is found. This relation is plotted in Fig. 6. In addition Fig. 6 shows the position of maximum temperature in the slab and the relative stress amplitude at the bonded surface.

The solution clearly shows resonance and antiresonance for $f$ values near multiples of $\pi / 2$. A variation in the boundary temperatures has only a small influence on the values of amplitude and frequency at resonance. The excitation amplitude $t$ needed to reach the melting temperature inside the slab increases by approximately $5 \%$ when the temperature $\theta_{s}$ at the wear surface is decreased from 0.7 to 0.6 (for Nylon 6.6 this is a temperature decrease of $53 \mathrm{~K}$ ). At resonance the shear stress amplitude increases from the excited to the bonded surface. Since the modulus is assumed to be constant 
(a)

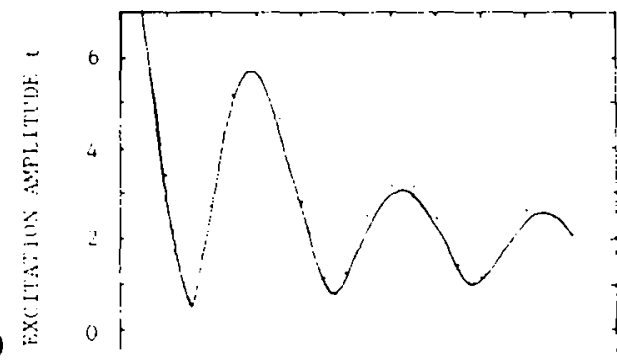

(b)

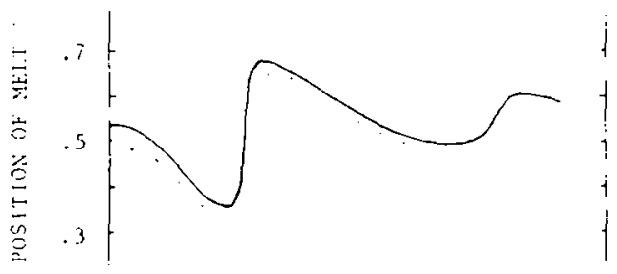

(c)

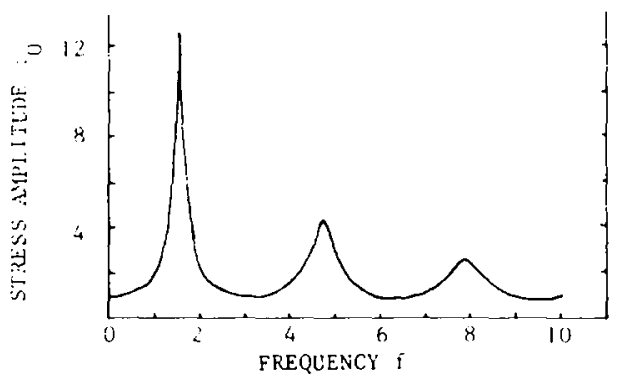

Fig. 6. Results of the constant $G$ model us. frequency for (a) the excitation amplitude $t$, (b) the position of maximum temperature $\xi$ and (c) the relative stress amplitude $\tau_{0}$ at the bonded surface $\left(\delta=0.1 ; \theta_{0}=0.6 ; \theta_{\mathrm{s}}=0.7 ; \cdot, \theta_{\mathrm{s}}=0.6\right)$.

throughout the slab, the maximum temperature is shifted towards the bonded surface.

For the first minimum in the $t-f$ curve (resonance), the distributions of the temperature and of the modulus and the argument of the complex shear stress in the slab are shown in Fig. 7. Figure 8 shows the effect of the loss tangent on the frequency, the amplitude and the position of maximum temperature, again at resonance. Under these conditions the product of the loss angle and the relative stress amplitude at the bonded surface is nearly constant. With increasing loss angle the resonant frequency decreases, the excitation amplitude increases and the position of maximum temperature is shifted slightly towards the excited surface of the slab.

To investigate the effect of the temperature dependence of the modulus, the system of equations (1) - (5) was numerically solved using a multiple-shooting method. The boundary conditions are the same as in the previous solution and the modulus was assumed to be a hyperbolic function of the temperature: 
(c)

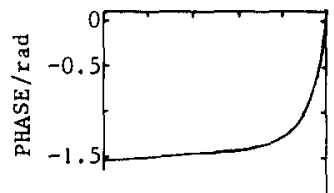

(b)

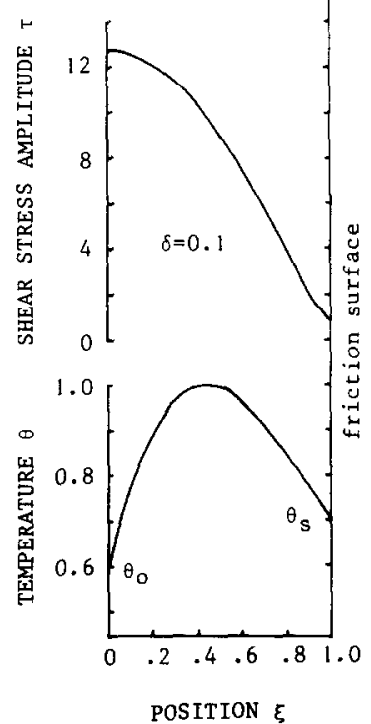

(a)

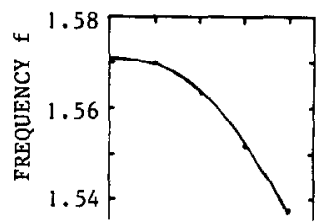

(b)

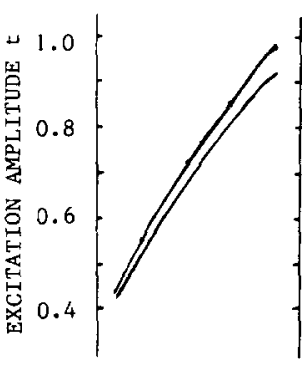

(c)

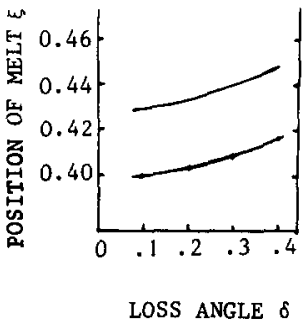

Fig. 7. The distribution at resonance of (a) the temperature, (b) the shear stress amplitude and (c) the phase shift in the shear stress $(\delta=0.1)$.

Fig. 8. The influence of the loss angle at resonance on (a) the frequency, (b) the excitation amplitude and (c) the position of maximum temperature $\left(\theta_{0}=0.6 ;-, \theta_{s}=0.7\right.$; $\rightarrow, \theta_{\mathrm{s}}=0.6$ ).

$G=0.8\left(\frac{1}{\theta}-1\right) \quad \mathrm{GPa}$

The two solutions are compared in Fig. 9.

The relation between excitation amplitude and frequency, resulting in a maximum temperature equal to the melting temperature, is similar in both solutions. For a temperature-dependent modulus the minimum at resonance seems to become less pronounced. Further analysis of the data showed that the position of maximum temperature was shifted towards the friction surface for a temperature-dependent modulus. This is in accordance with the observations from the "constant $G$ " model. Near the excited surface, the vibration amplitude will be smaller since $G$ increases with decreasing temperature. Consequently, the change in the stress amplitude is smaller and the maximum temperature shifts towards the friction surface. In order to produce better predictions regarding the position of maximum temperature, the numerical calculations should be extended and the variation in the loss angle with temperature should be included. 


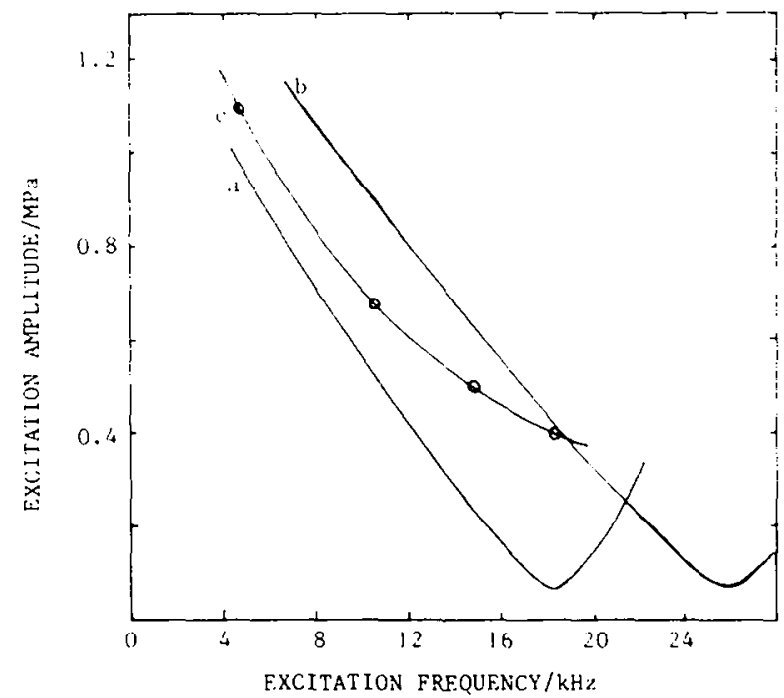

Fig. 9. Comparison between the numerical calculations (curve $\mathrm{c}$ ) and the constant $G$ model (curves a and b) $\left(T_{0}=318 \mathrm{~K}, T_{\mathrm{s}}=371 \mathrm{~K}, T_{\mathrm{m}}=530 \mathrm{~K}, d=4 \mathrm{~mm}\right)$ : curve a, $G=0.1$ $\mathrm{GPa}$; curve b, $G=0.2 \mathrm{GPa}$; curve c, $G=G(\theta), \delta=0.1$.

It should be noted that the solutions presented are based on the assumption of thermal equilibrium. In practice, the phenomenon will have a transient character. The resonant frequency will shift to lower values when the temperature distribution develops, and the vibrations will collapse when the melting temperature is reached inside the polymer.

\section{Experimental observations and discussion}

\subsection{General observations}

In Fig. 1 three differently shaped specimens of nylon are shown. These specimens were used in dry sliding experiments against steel on different machines, and all show one or more protrusions at a distance of $0.5-1.5 \mathrm{~mm}$ below the sliding surface. This, however, is not typical.

On all three tribometers vibrations were observed at frequencies between 10 and $100 \mathrm{kHz}$. A systematic investigation, however, into the relation between vibrations and subsurface recrystallization was performed only on the PS II tribometer. Unfortunately, the search for a criterion for subsurface changes during the sliding process has not been successful. Obviously, vibrations do not necessarily lead to temperatures inside the polymer high enough to initiate melting and subsequently to cause recrystallization. Also melting does not necessarily lead to protrusions. The prevailing conditions during sliding experiments, where subsurface recrystallization was observed afterwards, are described in Section 4.2.

Subsurface recrystallization and flow is illustrated in Figs. 2 and 10 12. Figure 10 shows a photomicrograph of the surface of a Nylon 6.6 


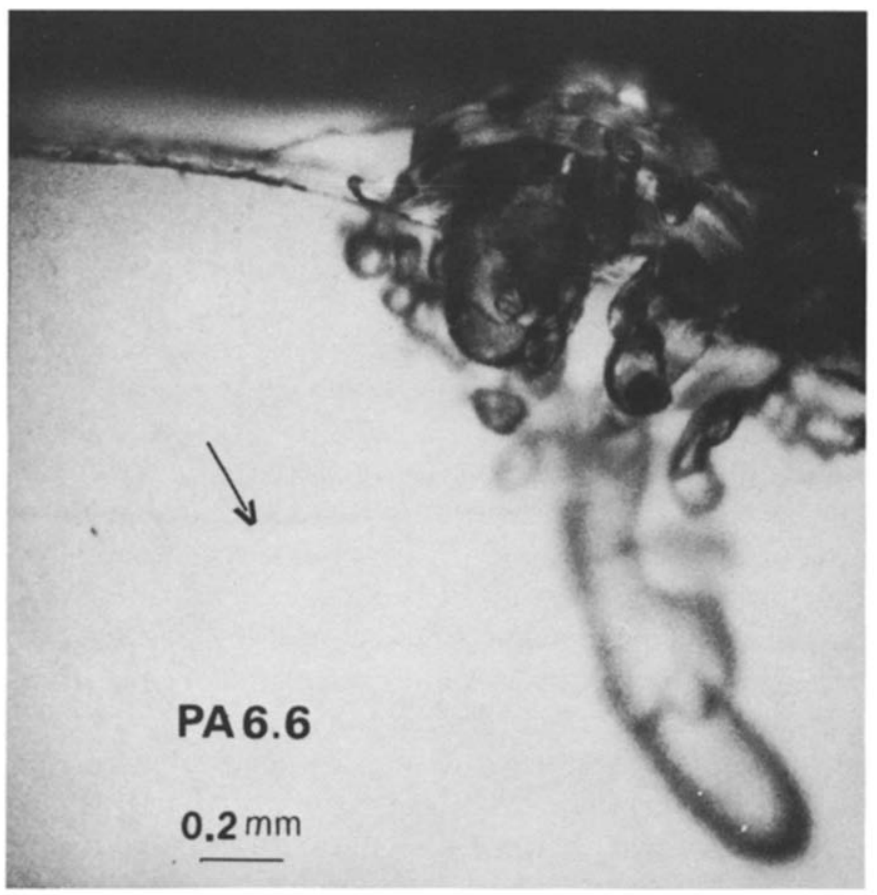

Fig. 10. Wear surface of a Nylon 6.6 pin near the edge.

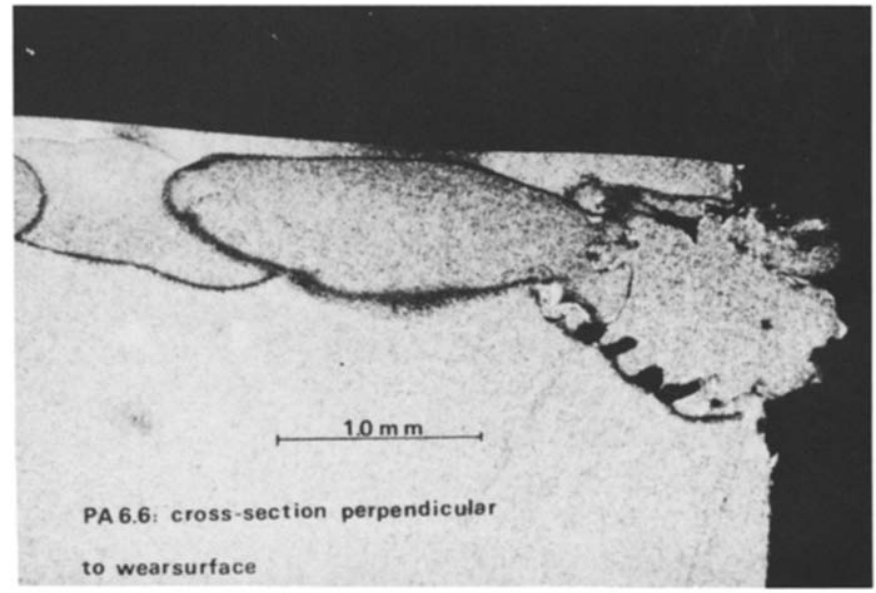

Fig. 11. A slice of Nylon $6.6(20 \mu \mathrm{m})$ showing repeated recrystallization (cross section perpendicular to the wear surface).

specimen. The side illumination makes the protruded material and the voids below the surface clearly visible. Back-transferred nylon can be seen on the surface.

Recrystallization is shown in Fig. 2. This figure shows a thin slice of Nylon 6.6 perpendicular to the wear surface and parallel to the friction 


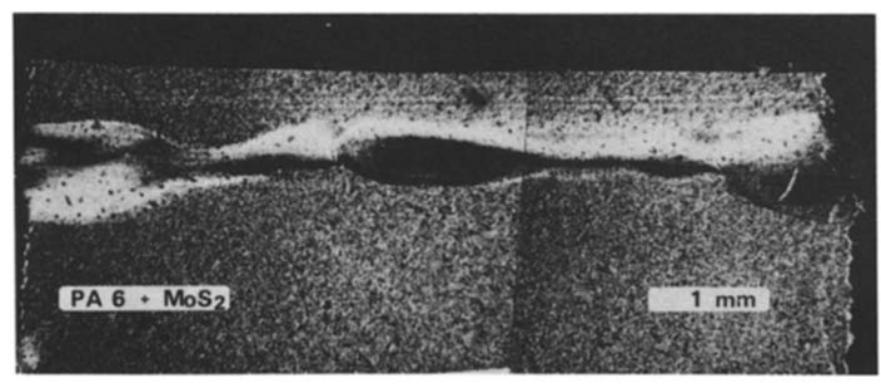

Fig. 12. A slice of Nylon 6 with $\mathrm{MoS}_{2}$ filler $(20 \mu \mathrm{m})$ showing subsurface recrystallization.

force. It was taken through a protrusion near the leading edge of a specimen. The friction force on the specimen acted from left to right as can be seen from the permanent deformation at the edge. The molten areas are shaped like ellipsoids, having their longer axis parallel to the force of friction. After recrystallization the spherulites have the same dimensions as before melting, except near the boundaries. Here the spherulites are much smaller, indicating tensile stresses in the material. The surface depression, probably resulting from the wear of a local bulge due to thermal expansion, should be noted. Further, it should be noted that material from the area on the right-hand side seems to have been squeezed into the larger area on the left-hand side. This is more clearly shown in Fig. 11. This photomicrograph shows essentially the same features as Fig. 2. The friction force in this picture, however, acts from right to left. Furthermore, it shows repeated recrystallization. Apparently, the material can experience repeated cycles of melting and recrystallization without extrusion of material. In fact this is the most common process, but its occurrence cannot be ascertained by visual inspection of the specimen. The scattering of visible light at the recrystallization boundaries is much too small to be detected.

A rather extreme case of subsurface recrystallization without the formation of a protrusion is shown in Fig. 12. This represents a slice of Nylon 6 filled with $\mathrm{MoS}_{2}$ again taken perpendicular to the wear surface and parallel to the friction force (acting from right to left). Recrystallization occurred throughout the sample, from the leading edge to the trailing edge. Finally, it should be noted that the wear surfaces in these photographs do not show any sign of melting or thermal degradation.

\subsection{Conditions favouring subsurface recrystallization}

In earlier work on the dry sliding friction of nylon on steel two types of frictional behaviour have been reported [11]. Vibrations and subsurface recrystallization have been found only in cases where type $B$ friction was dominant. The friction then rises sharply at temperatures above the glass transition temperature (Fig. 13).

A typical time-averaged vibration spectrum is shown in Fig. 14(a). The instantaneous spectrum (Fig. 14(b)) consists of a few sharp peaks. Variation in the spectrum is usually accompanied by changes in the friction force and 
in the appearance of the transfer film. A typical example of a cyclic variation in the friction is shown in Fig. 15.

When the friction starts to increase, the frequency spectrum shows one or two peaks near $20 \mathrm{kHz}$. The transfer film on the disc becomes very smooth. When the friction levels off, the transfer film becomes rougher and breaks up into small islands. Simultaneously the friction collapses and the peaks in the frequency spectrum disappear.

Although cyclic variations in the friction force were frequently observed at temperatures above the glass transition temperature, subsurface recrystallization was also found in cases where the friction force was almost constant, at constant load, speed and surface temperature of the disc. In the range $0.4-1.5 \mathrm{~m} \mathrm{~s}^{-1}$, the speed of sliding has no influence on the frequency of vibrations. At lower speeds, the dominant frequencies are below $18 \mathrm{kHz}$ and subsurface recrystallization is less frequently observed. The normal load

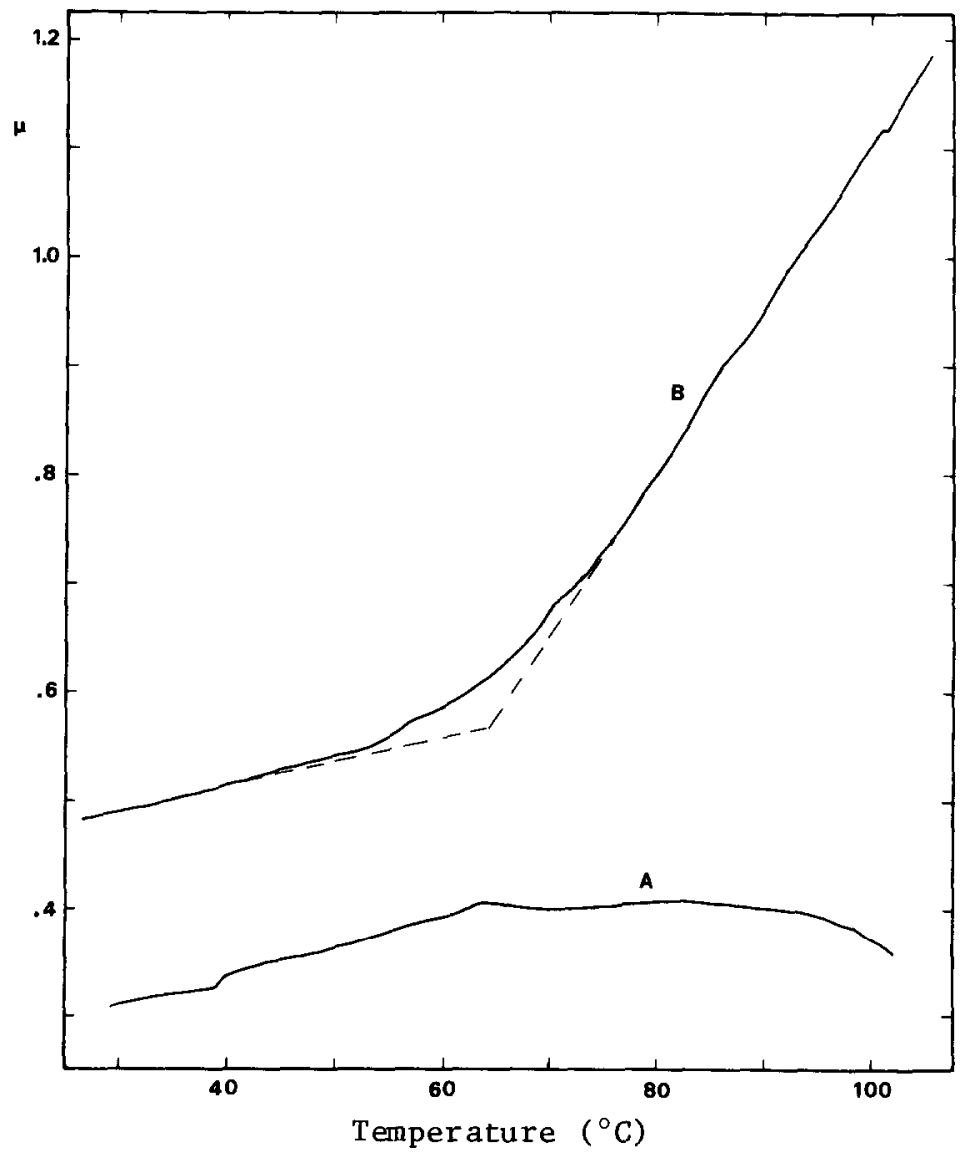

Fig. 13. Typical friction us. temperature curves for Nylon 6.6 on steel: curve $A$, steel surface turned on a lathe; curve $B$, steel surface finished by abrasion. 
and the surface temperature of the steel disc do not seem to have much influence on either vibrations or recrystallization.

The dominant peaks in the vibration spectrum observed during different experiments are shown as a function of the disc temperature in Fig. 16. The full curve in this figure represents the natural frequency calculated for a slab of $4 \mathrm{~mm}$ thickness having a uniform temperature throughout the slab

(a)

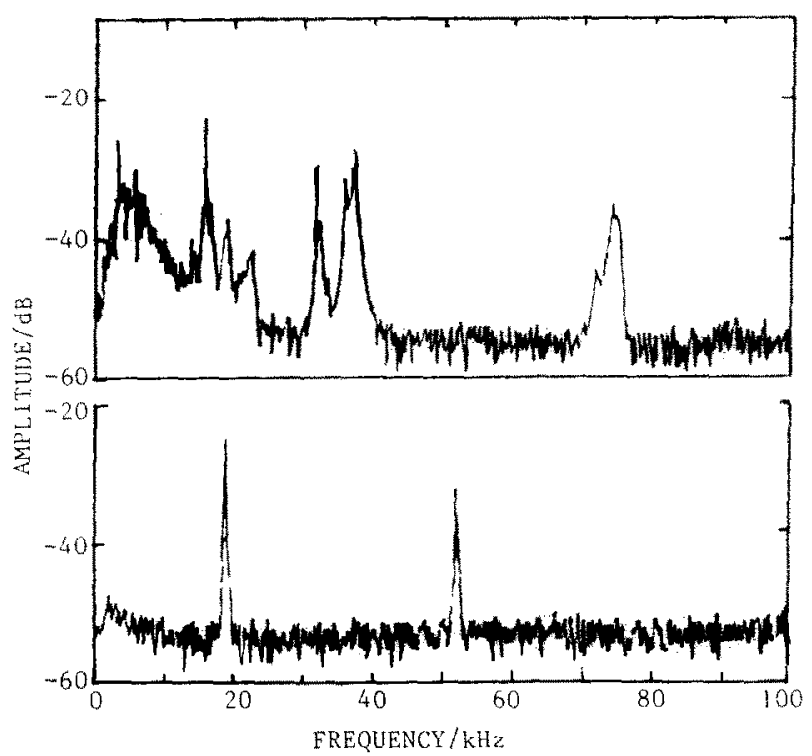

Fig. 14. Typical vibration spectra: (a) time-averaged spectrum; (b) instantaneous spectrum.

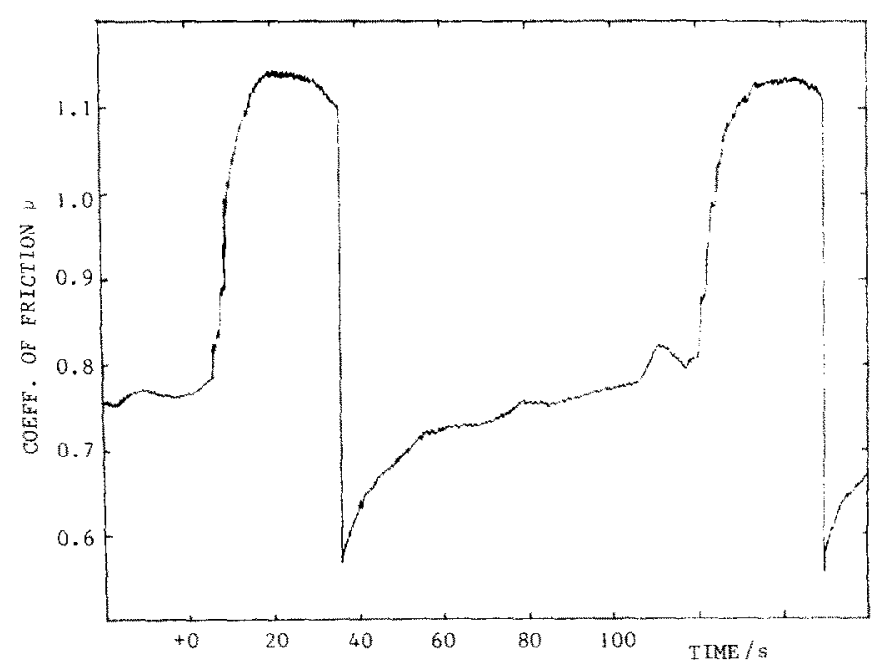

Fig. 15. Cyclic variation in the coefficient of friction (Nylon 6.6 on C45 steel; load, $50 \mathrm{~N}$; speed, $0.16 \mathrm{~m} \mathrm{~s}^{-1}$; temperature, $100^{\circ} \mathrm{C}$ ). 


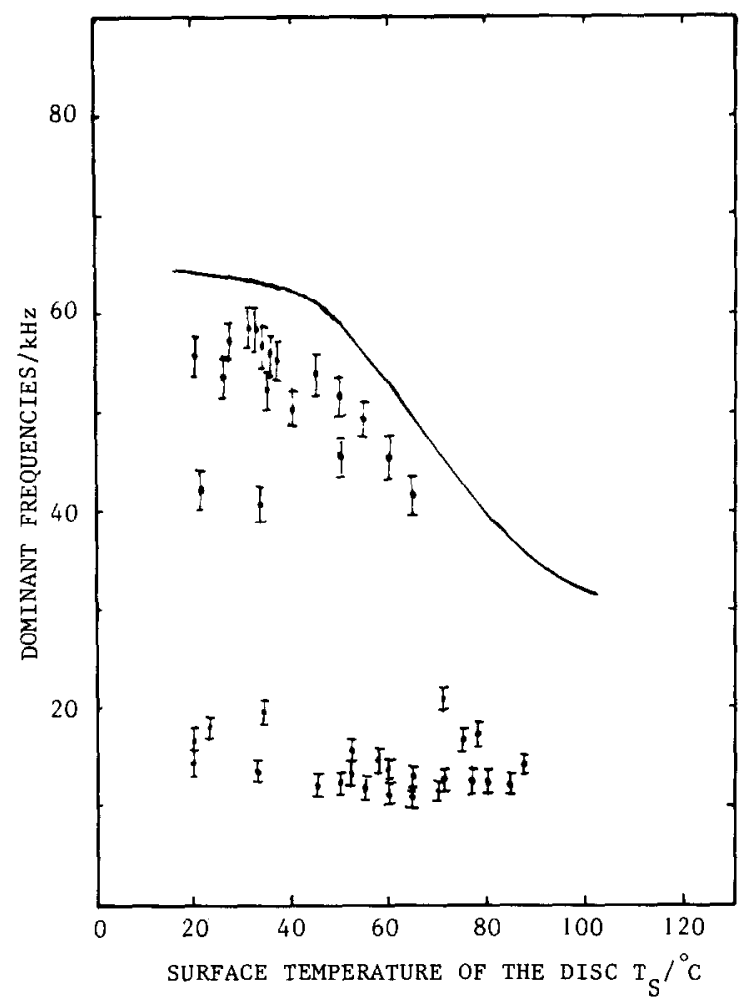

Fig. 16. Measured frequencies vs. surface temperature of the disc (Nylon 6.6).

equal to the surface temperature $T_{\mathrm{s}}$. The modulus is taken according to the full curve in Fig. 5 and the dimensionless frequency $f=\pi / 2$ (no damping). These results suggest that the high frequencies (above $30 \mathrm{kHz}$ ) are associated with resonance of the "cold" slab. Internal heating due to damping of these vibrations shifts the resonant frequency to a value between 15 and $20 \mathrm{kHz}$ which is in fair agreement with the calculated results shown in Fig. 9.

\subsection{Wear}

The wear of nylon on steel strongly depends on the surface temperature of the steel. In Fig. 17 results of previous measurements on Nylon 6.6 are shown [11]. The wear measured in the present experiments ranged from 1 to $10 \mathrm{mg}$, giving a wear depth of $20-200 \mu \mathrm{m}$. Specific wear rates are between $3 \times 10^{-6}$ and $13 \times 10^{-6} \mathrm{~mm}^{3} \mathrm{~N}^{-1} \mathrm{~m}^{-1}$. A direct comparison with Fig. 17 is not possible because in most cases the speed of sliding or the disc temperature was changed during the experiments. The specific wear rates given above are averaged values, but they are in good agreement with previous data.

In one experiment shear occurred over the whole cross section and a layer of $0.7 \mathrm{~mm}$ was lost from the pin, but generally no direct influence of vibrations or subsurface melting on wear was observed. The wear depth in 


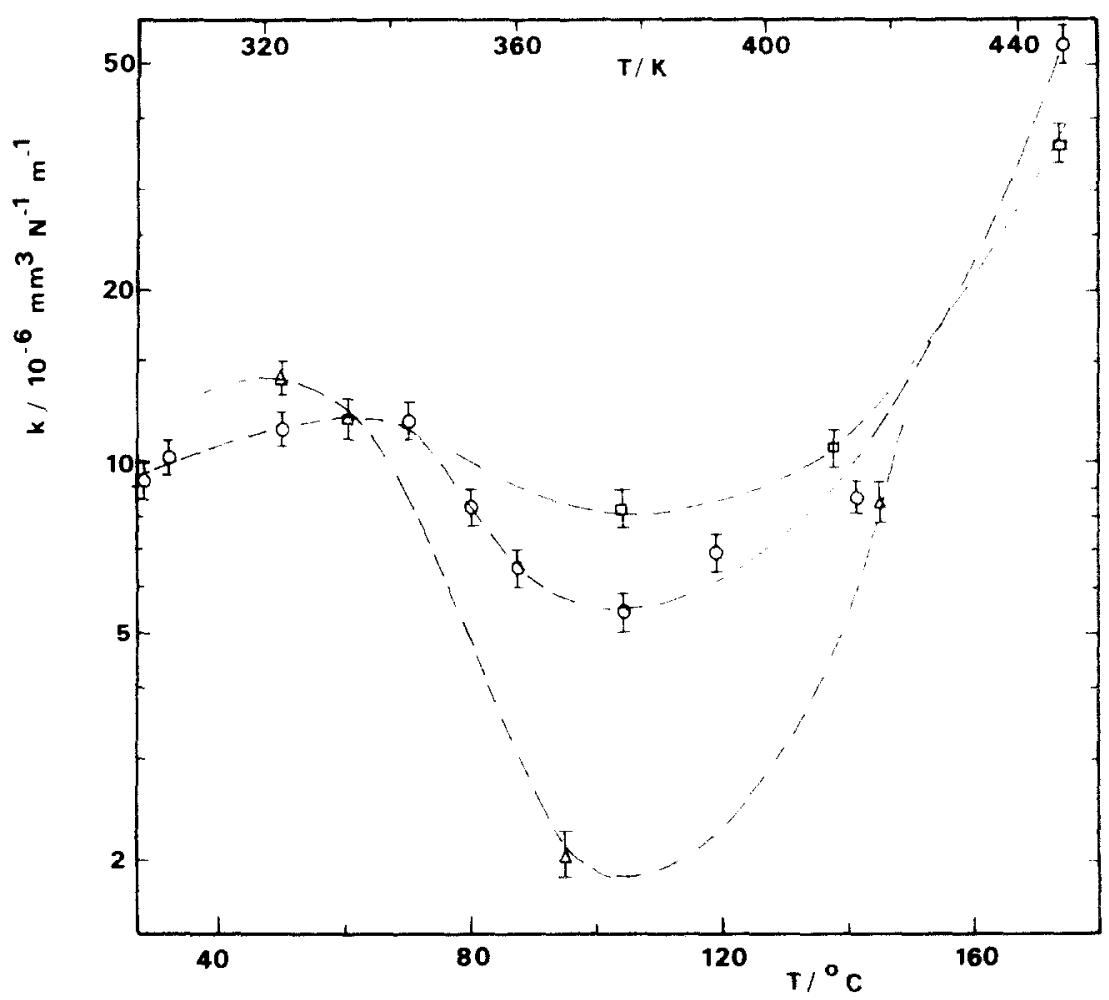

Fig. 17. Specific wear rate $v s$. disc temperature for various speeds (load, $50 \mathrm{~N}$ (1 MPa); $\left.R_{\mathrm{a}}=1.6 \mu \mathrm{m}\right): \square, 0.10 \mathrm{~m} \mathrm{~s}^{-1} ; 0,0.15 \mathrm{~m} \mathrm{~s}^{-1} ; \Delta, 0.30 \mathrm{~m} \mathrm{~s}^{-1}$.

these experiments is obviously too low, but after prolonged sliding the recrystallization boundaries would reach the surface and an increase in wear rate could be expected. Indirect effects could also occur as a result of thermal expansion of the polymer during internal heating, for instance when the clearance in a bearing is not large enough.

\section{Conclusions}

The phenomenon of subsurface recrystallization in nylon is described and explained as a result of subsurface melting. The heat needed for melting is provided by the damping of a resonating shear wave inside the polymer. This shear wave is excited by the friction process, but the precise excitation mechanism still needs to be explained. Frequencies, calculated from a thermomechanical vibration model, under rather simplifying assumptions with regard to the mechanical properties, are in reasonable agreement with measured vibration frequencies. For a slab of Nylon 6.6 having a thickness of $4 \mathrm{~mm}$, this frequency is near $20 \mathrm{kHz}$ when the maximum temperature equals the melting temperature. 
For these thick slabs no direct influence on the wear rate was detected. The model predicts the resonant frequency to be inversely proportional to the thickness and the excitation amplitude to be inversely proportional to the square root of the thickness. Thus for thin layers the occurrence of resonance could easily lead to destruction of the layer.

Recrystallization and the formation of protrusions can be explained as follows. Any irregularity in the distribution of the surface forces will result in a locally increased thermal expansion perpendicular to the wear surface and thus in a concentration of the surface forces towards one spot. When melting occurs, the molten material may eventually break away through the wall of the specimen. Next the surface forces will disperse, the molten material cools down and recrystallizes and the same process may repeat at a different spot. If the molten polymer cannot break away, either through the wall or into a void, the surface spot wears off until the load concentration disperses, leaving an indentation in the friction surface of the polymer after recrystallization of the melt.

\section{Acknowledgments}

Thanks are due to Mr. L. Vogels for performing most of the experimental work and to the companies DSM and Polypenco BV for providing the polymers.

\section{References}

$1 \mathrm{H}$, von Helmholz, Die Lehre von der Tonempfindungen als physiologische Grundlage für die Theorie der Musik, 1862.

2 H. Blok, Fundamental aspects of boundary friction, J. Soc. Automot. Eng., 46 (1940) 275.

3 C. A. Brockley, R. Cameron and A. F. Potter, Friction induced vibrations, J. Lubr. Technol., 89 (1967) $101 \cdot 108$.

4 Plato, Timaeus.

5 B. Bhushan, Stick-slip induced noise generation in water lubricated compliant rubber bearings, J. Lubr. Technol., 102 (1980) $201-212$.

6 E. H. Lee, Thermoviscoelasticity, Proc. IUTAM Symp. on the Mechanics of ViscoElastic Media and Bodies, 1974, Springer, Berlin, 1975.

7 E. C. Ting, Dissipation function of a viscoelastic material with temperature dependent properties, J. Appl. Phys., 44 (11) (1973) 4956 - 4960.

8 N. C. Huang and E. H. Lee, Thermomechanical coupling behavior of viscoelastic rods subjected to cyclic loading, J. Appl. Mech., (March 1967) 127 - 132.

9 E. C. Ting, Thermomechanical coupling effects in the longitudinal oscillations of a viscoelastic cylinder, J. Acoust. Soc. Am., 52 (3) (1972) 928 - 934.

10 A. C. Pipkin, Lectures on Viscoelasticity Theory, Springer, New York, 1972.

11 P. H. Vroegop, H. H. Vermeulen and R. Bosma, The influence of temperature, speed and roughness on the dry sliding friction and wear of nylon 6.6 against steel. In $D$. Dowson, C. M. Taylor, M. Godet and D. Berthe (eds.), Friction and Traction, Proc. 7th Leeds-Lyon Symp. on Tribology, Leeds, September 9-12, 1980, Westbury House, Guildford, 1980, pp. $93-98$.

12 A. W. J. de Gee, The friction of gold-silver alloys against steel, Wear, 8 (1965) 121 . 132.

13 D. W. van Krevelen, Properties of Polymers, Elsevier, Amsterdam, 2nd edn., 1976. 